\title{
Triple, Mutually Orthogonal Cycloadditions Through the Design of Electronically Activated SNO-OCTs
}

\author{
Yun Hu, ${ }^{\dagger}+$ Jessica M. Roberts, ${ }^{\dagger}$, Henry R. Kilgore,${ }^{\S, \dagger}$ Amirah S. Mat Lani, ${ }^{\dagger}$ Ronald T. Raines, ${ }^{*, \S}$ and \\ Jennifer M. Schomaker*,† \\ ${ }^{1}$ Department of Chemistry, University of Wisconsin, Madison, Wisconsin 53706, United States \\ ${ }^{2}$ Department of Chemistry, Massachusetts Institute of Technology, Cambridge, Massachusetts 02139, United States
}

\begin{abstract}
Interest in mutually exclusive pairs of bioorthogonal labeling reagents continues to drive the design of new compounds capable of fast and predictable reactions. The ability to easily modify heterocyclic strained cyclooctynes containing sulfamate backbones (SNO-OCTs) enables electronic tuning of the relative rates of reactions of SNO-OCTs in cycloadditions with Type I-III dipoles. As opposed to optimizations based on just one specific dipole class, the electrophilicity of the alkynes in SNO-OCTs can be manipulated to achieve divergent reactivities and furnish mutually orthogonal dual ligation systems. Significant rate enhancements for reactions of a difluorinated SNO-OCT derivative compared to the parent scaffold were noted, with the second-order rate constant in cycloadditions with diazoacetamides exceeding $1 \mathrm{M}^{-1} \mathrm{~s}^{-1}$. Computational and experimental studies were employed to inform the design of triple ligation systems that encompass three orthogonal reactivities. Finally, polar SNO-OCTs are rapidly internalized by mammalian cells and remain functional in the cytosol for live-cell labeling, highlighting their potential for diverse in vitro and in vivo applications.
\end{abstract}

\section{INTRODUCTION}

Bioorthogonal chemistry represents a powerful strategy to monitor individual molecules in biological systems; indeed, within the past two decades, a multitude of reactions have been developed to fulfill this function. ${ }^{1}$ Nonetheless, a larger challenge involves the identification of mutually orthogonal pairs of bioorthogonal reagents with the potential to simultaneously observe the behavior of multiple biomolecules in a cell. ${ }^{2}$ The ready availability of convenient, inexpensive, and electronically tunable "orthogonal bioorthogonal" reagent pairs remains a topic of intense interest, as new labeling tools are critical in efforts to push the boundaries of our understanding of complex biological systems. ${ }^{2 \mathrm{~d}, 3}$

The 1,3-dipolar cycloadditions are popular reactions in bioorthogonal chemistry, particularly strain-promoted azidealkyne cycloadditions (SPAAC; Figure 1a). ${ }^{1 \mathrm{~b}, 3 \mathrm{~b}, 4}$ Bertozzi and co-workers addressed issues with the slow kinetics of unfunctionalized cyclooctynes by electronically activating the alkyne via installation of fluorine atoms at the propargyl carbon. Moving from monofluorinated (MOFO) to difluorinated alkyne (DIFO) resulted in nearly two orders of magnitude increase in the rate of reaction with $\mathrm{BnN}_{3}$, from 0.0043 to $0.076 \mathrm{M}^{-1} \mathrm{~s}^{-1}$. The popular bioorthogonal reagent $\mathrm{DBCO}$ utilizes benzene rings fused to the cycloalkyne to further accelerate the reaction with $\mathrm{BnN}_{3}$; however, further electronic tuning of this scaffold is challenging, due to limited derivatization sites. ${ }^{6}$ Other reported modifications to the parent compound yield reaction rates with $\mathrm{BnN}_{3}$ spanning less than an order of magnitude $\left(0.31-0.90 \mathrm{M}^{-1} \mathrm{~s}^{-1}\right){ }^{7}$ In addition to the lack of modularity, the strain-activated nature of this alkyne leads to issues with stability and chemoselectivity, limiting opportunities to develop mutually orthogonal reactions involving DBCO derivatives. ${ }^{8}$

Recently, we developed a unique class of strained cycloalkynes containing $S$-, $N$-, and $O$-heteroatoms embedded in the ring (SNO-OCTs). ${ }^{9}$ Our previous kinetic studies of the sulfamate-containing SNO-OCTs 1-2 (Figure 1a) show secondorder kinetic rate constants $\left(k_{2}\right)$ with $\mathrm{BnN}_{3}$ ranging from 0.0014 to $0.087 \mathrm{M}^{-1} \mathrm{~s}^{-1}$, with polar protic solvents further enhancing the rate of 2 to $0.13 \mathrm{M}^{-1} \mathrm{~s}^{-1} .^{1 \mathrm{k}, 9}$ Despite the relaxed alkyne angles and reduced ring strain in $\mathbf{1}-\mathbf{2}$, these SNO-OCTs exhibit a significant rate-increase for cycloadditions, comparable to strain-activated DIBONE and DBCO. Density functional theory (DFT) calculations indicated these SNO-OCTs benefit from unique electronic activation of the alkyne via tailored anomeric effects and hybridization, resulting in robust stability at high $\mathrm{pH}$ and in the presence of glutathione. ${ }^{2 \mathrm{~b}, 9}$ Furthermore, the presence of the sulfonyl group increases the polarity in the transition state, which has been shown to enhance the rates of cycloadditions. ${ }^{9}$

The tunability of SNO-OCTs begs the question of whether these scaffolds can be tailored for divergent reactivity with diverse coupling partners. The Prescher group developed several mutually orthogonal dual ligation systems, parti-cularly a series of cyclopropenes that could be sterically tuned to achieve mutually orthogonal Type I and Type III cycloadditions (Figure 1b). ${ }^{2 \mathrm{c}-\mathrm{d}, 3 \mathrm{c}}$ Sterically encumbered 3,3-cyclopropenes prefer nitrile imines, while shifting the bulk in 1,3cyclopropenes favors reaction with tetrazines. Similarly, Franzini and co-workers designed sterically differentiated isonitriles, which display chemoselective reaction with various tetrazine derivatives. ${ }^{10}$ Increasing the steric bulk on the tetrazine resulted in successful dual orthogonal labeling of a protein in the presence of trans-cyclooctene (TCO) and DBCO. Computational analysis indicated bulky tetrazines benefit from dispersion forces that favor reaction with isonitriles. Though steric tuning has proven successful for multiple ligation systems, the ability to tune SNO-OCT alkyne electronics in a predictable manner could tailor the chemoselectivity to achieve mutual orthogonality across diverse coupling partners without rate reductions often seen with steric-driven strategies. 


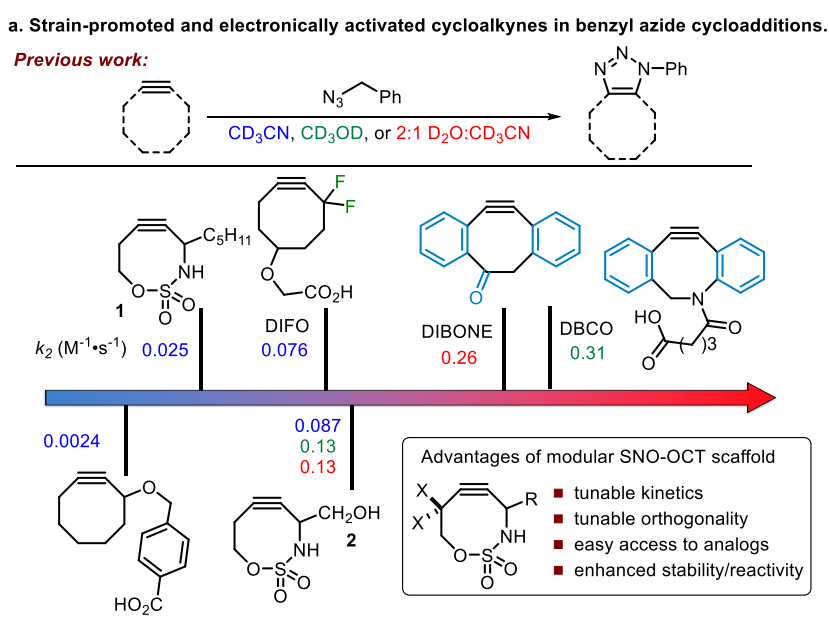

b. Sterically controlled chemoselectivity to achieve mutual orthogonality.

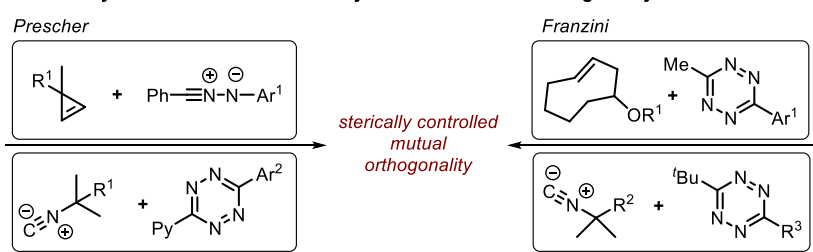

c. Tuning alkyne electronics in SNO-OCTs to afford divergent reactivity. This work:

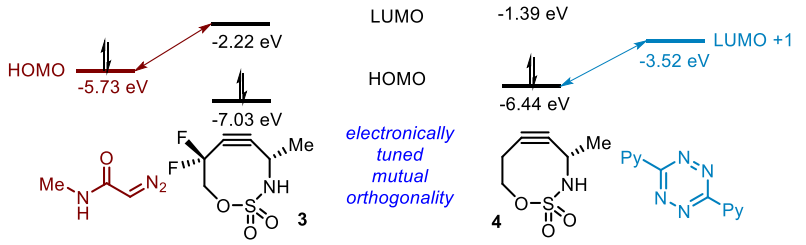

Figure 1. Previous work and design of new mutually "orthogonal bioorthogonal" reactions for multicomponent labeling.

This paper reports on the electronic tuning of SNO-OCTs to achieve chemoselective reactions with Type I, Type II, and Type III dipoles with fast kinetics. Frontier molecular orbital (FMO) and natural bond orbital (NBO) analyses were used to rationalize how derivatization influenced alkyne electronics, ${ }^{11}$ while distortion/interaction analysis shed insight into how electronics and strain impacted reactivity trends. Computational insights guided the identification of potentially orthogonal SNO-OCT/dipole pairs; for example, the electron-deficient alkyne of difluorinated SNO-OCT 3 (dF-SNO-OCT) was expected to display divergent reactivity relative to the parent 1 (Figure 1c). Successes in developing new dual and triple ligation systems highlight the versatility of SNO-OCTs for chemoselective reactions with diverse dipoles by modifying the alkyne electronics. Finally, the use of a novel triple ligation system for protein labeling is demonstrated, and the application of SNO-OCTs as efficient cell-surface visualization agents is described.

\section{RESULTS AND DISCUSSION}

Computational Methods Development to Predict SNO-OCT Cycloaddition Reaction Rates. To facilitate rational design of promising SNO-OCT derivatives (see Figure S1), we sought to develop a computational approach capable of accurately predicting reaction rates and reactivity trends. As numerous SNO-OCT derivatizations can be envisaged, DFT calculations were needed to rapidly identify potential mutually orthogonal reactions and streamline experimental efforts. Initial computations conducted at the M06-2X level of theory ${ }^{12}$ with a $6-311++G(d, p)$ basis set using Gaussian $16^{13}$ showed predicted rates that deviated significantly from experimental values (Table 1). For example, previous studies showed that 1 reacts with $\mathrm{BnN}_{3}$ in $\mathrm{CH}_{3} \mathrm{CN}$ with $k_{2}=2.60 \times 10^{-2} \mathrm{M}^{-1} \mathrm{~s}^{-1}$ (Table 1, entry 1); however, DFT calculations predicted a $k_{2}$ value that was $\sim 3$ orders of magnitude lower, at $6.21 \times 10^{-5} \mathrm{M}^{-1} \mathrm{~s}^{-1}$. M06$2 \mathrm{X}$ has been previously shown to overestimate activation barriers, with Houk applying a correction factor to computed values to bring them into better quantitative agreement. ${ }^{14}$ Unfortunately, this correction factor is based on experimental second-order rate constants for similar cycloadditions; because our goal was to utilize computational methods to predict relative rates of derivatized SNO-OCT scaffolds, a different level of theory was required. Zuilhof also reported that M06-2X overestimates activation barriers in strain-promoted oxidationcontrolled quinone (SPOCQ) cycloadditions and found that switching to the B97D functional gave calculated rates that aligned better with experimental data. ${ }^{15} \mathrm{SNO}-\mathrm{OCT}$ rates were calculated at the B97D level of theory using a Conductor-like Polarizable Continuum Model for the solvent (see the SI for details). ${ }^{16}$ B97D improved the agreement between computed and experimental $k_{2}$ values (within $\sim 1$ order of magnitude) and gave more accurate $k_{\text {rel }}$ ratios for the experimental:predicted rates in reactions of 1/4:2 with the corresponding azides. In contrast, calculations at the M06-2X level of theory predict a slower rate of reaction for 2 with $\mathrm{BnN}_{3}$ as compared to 4 (Table 1 , entry 2), which is not manifested experimentally. Thus, the B97D functional furnishes calculated rates that are much more predictive of experimental values than does M06-2X. Accordingly, further computational analyses were conducted utilizing the B97D level of theory to predict potential mutual orthogonality of SNO-OCT derivatives.

Table 1. Experimental and calculated $k_{2}$ values for cycloadditions of SNO-OCTs with alkyl azides.

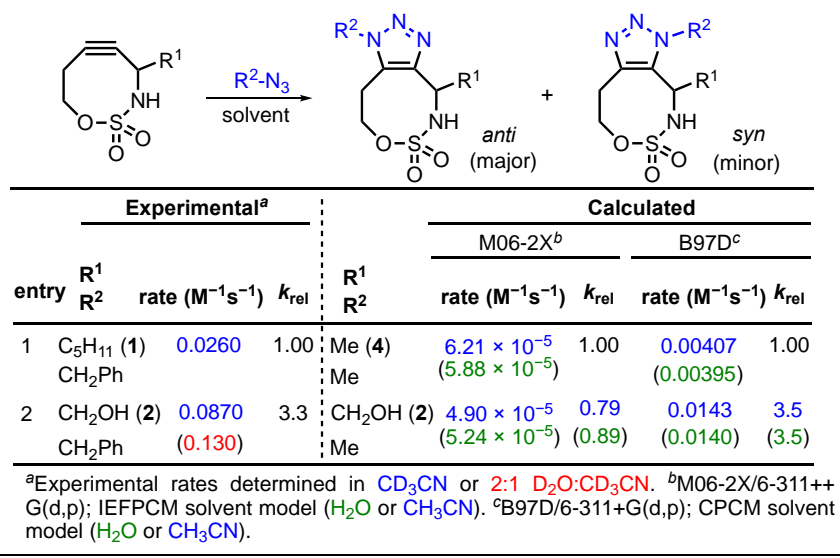

SNO-OCT derivatization to increase reaction rates. Our ability to readily change the substitution pattern of the SNOOCT scaffold stimulated efforts to electronically tune the alkyne to: 1) improve rates of dipolar cycloadditions, 2) achieve mutually exclusive orthogonal reactivities of SNO-OCTs with Type I-III dipoles in [3+2] and [4+2] cycloadditions, and 3) highlight the ability to install multiple functional handles for appending dyes and reporter molecules to SNO-OCTs for biological applications. As Type I-III cycloadditions can be designed based on FMO considerations, the potential for 
mutually orthogonal reactivity may be realized if effective orbital matching can be achieved. We hypothesized an electronpoor, electrophilic SNO-OCT derivative might engage in chemoselective reaction with a nucleophilic diazoacetamide dipole; furthermore, the reaction should be orthogonal to an inverse electron-demand Diels-Alder (IEDDA) reaction between a more electron rich SNO-OCT and dipyridyl tetrazine. Azide cycloadditions were also studied computationally to determine if a triple SNO-OCT ligation system might transcend all three dipole classes.

NBO analyses ${ }^{11}$ of geometry-optimized SNO-OCTs $\mathbf{2 - 4}$ in water were used to study the effects of derivatization on alkyne electronics. Multiple orbital interactions exist within the SNOOCT scaffold; these may differ greatly in their contributions to the alkyne electronics, depending on the nature of the substitution in the alkyl side chain or the propargylic substitution (Figures 2a and S1-2). The predicted SNO-OCT alkyne electrophilicities were determined based on the $2^{\text {nd }}$-order perturbation energies of the relevant orbital interactions (Figure 2b, Table S1-1). As might be expected, SNO-OCT 4 and its hydroxylated counterpart $\mathbf{2}$, which lack fluorine at the propargylic site, contain electron-rich alkynes. Introduction of a single propargyl fluorine (3a) yields a less electron-rich alkyne, while a second fluorination to furnish dF-SNO-OCT 3 contains the most electrophilic alkyne of the series. It is important to note the calculations suggest modification of the $\mathrm{R}$ group in $\mathbf{2}$ and $\mathbf{4}$ minimally impacts the $\pi$ system of the alkyne, whereas modifications at the propargylic site adjacent to the reactive center lead to dramatic changes in electrophilicity.

Identifying Potential Orthogonal Reactivity via Computed Reaction Rates. While qualitative predictions of SNO-OCT/Type I-III dipoles likely to yield mutually orthogonal systems might be gleaned from Figure 2, reaction modeling could help select optimal cycloaddition partners. Transition state (TS) modeling of reactions between SNOOCTs 2-4 and an example from each dipole class (Type I, methyl diazoacetamide D1; Type II, methyl azide D4; Type III, dipyridyltetrazine D3) were carried out to assess potential orthogonality (Figure 3). Cycloadditions of nucleophilic Type I dipoles should be accelerated with electron-deficient alkynes, due to a lowering of the LUMO and a reduced FMO energy gap. ${ }^{17}$ Indeed, difluorination in $\mathrm{dF}-\mathrm{SNO}-\mathrm{OCT} 3$ was predicted to give a $\sim 300$-fold increase in reaction with D1, as compared to 2 and 4 (Figure 3a,e). Distortion/ interaction ${ }^{18}$ or activation strain $^{19}$ analysis on calculated transition state models shed further insight into the key features of the SNO-OCT driving reactivity. Computed reactivities of $\mathbf{2 - 4}$ in reactions with methyl diazoacetamide correlate with the total distortion energy required to reach the transition state geometry (Figure 3d, $E_{\mathrm{dis}, \mathrm{SNO}-\mathrm{OCT}}$ and $E_{\mathrm{dis,dipole}}$; Figure S1-4); TS2a and TS4a require further distortion of the dipole, as evidenced by the reduced bond angles $\left(1.3-2.1^{\circ}\right.$ contraction, Figure $\left.3 \mathrm{a}\right)$. Furthermore, $\mathrm{dF}$ SNO-OCT 3 benefits from pre-distortion of the alkynyl bond angles and requires only a $6.34^{\circ}$-bond contraction of the distal alkynyl angle, whereas 2 and $\mathbf{4}$ require over $8.5^{\circ}$. These reactions also show excellent correlation to the interaction energy (Figure 3d, $E_{\text {int }}$ ) and the calculated alkyne electrophilicity, with dF-SNO-OCT 3 showing the largest a. Orbital interactions leading to changes in alkyne electronics

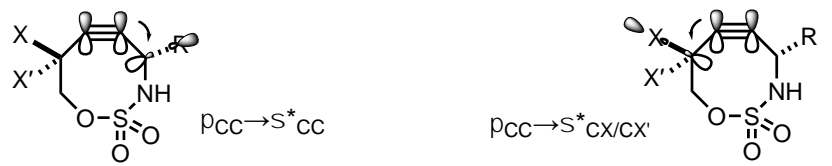

b. Fluorination leads to substantial alkyne electrophilicity enhancement increasing electrophilicity of the alkyne*

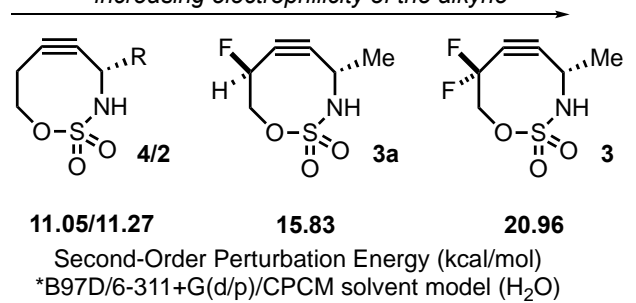

Figure 2. NBO analysis of orbital interactions contributing to predicted alkyne electrophilicity of SNO-OCTs. ${ }^{11}$

stabilizing interaction energy between coupling partners $(+3.1$ $\mathrm{kcal} \cdot \mathrm{mol}^{-1}$ relative to 4). This distinction is explicable by FMO considerations, as the most electron-deficient alkynes benefit from lower-lying LUMO levels; the presence of the propargylic fluorines stabilizes the $\operatorname{LUMO}(\mathbf{3},-2.22 \mathrm{eV}$ compared to 2, $-1.45 \mathrm{eV} ; 4,-1.39 \mathrm{eV}$; Figure S1-3). Overall, Type I cycloadditions appear to be influenced by both strain and electronic components of the SNO-OCT (Figure S1-4).

Type III cycloadditions, as exemplified by IEDDA, should display faster reaction kinetics with electron-rich alkynes, due to destabilization of the HOMO. Previous computational studies on these systems investigated the transition states for either the cycloaddition ${ }^{20}$ alone or both the cycloaddition and $\mathrm{N}_{2}$ evolution. ${ }^{21}$ The cycloaddition TS is expected to be the kinetically relevant barrier, and the values reported herein correspond to the cycloaddition TS. Dipyridyl tetrazine D3 (Figure 3b) was modeled, as the electron-withdrawing pyridyl groups enhance cyclo-addition rates due to LUMO+1 stabilization of the dipole. ${ }^{20}$ As predicted, electron-rich SNOOCTs 2 and 4 (HOMO levels $-6.42 \mathrm{eV}$ and $-6.44 \mathrm{eV}$ respectively) exhibit the fastest kinetics, whereas $\mathrm{dF}-\mathrm{SNO}-\mathrm{OCT}$ 3 shows significantly lower reactivity due to HOMO stabilization $(-7.03 \mathrm{eV})$. Furthermore, dipole distortion energy for reaction with $\mathrm{dF}-\mathrm{SNO}-\mathrm{OCT} \mathbf{3}$ is elevated relative to $\mathbf{2}$ and 4, likely due to the $0.2 \AA$ shortening of the distal $\mathrm{C}-\mathrm{C}$ bond, yielding increased steric interactions with the elongated $\mathrm{C}-\mathrm{F}$ bonds $(\sim 0.3 \AA)$. Hydroxylated SNO-OCT 2 was predicted to display a five-fold rate enhancement as compared to 4 . Reactivity trends showed moderate-to-excellent correlation to both distortion and interaction energies (Figures 3d and S1-5)

Based on reactions of SNO-OCTs with Type I and III dipoles, the potential for mutually orthogonal double ligation appeared promising. The next challenge was to identify a third orthogonal reaction, ideally between a SNO-OCT and a Type II dipole. Thus, reactions of 2-4 with azide D4 were modeled for comparison (Figure 3c). In Type II cycloadditions, reaction can occur via $\mathrm{HOMO}_{\text {dipolarophile }}-\mathrm{LUMO}_{\text {dipole }}$ or $\mathrm{HOMO}_{\text {dipole }}-$ $\mathrm{LUMO}_{\text {dipolarophile }}$ interactions, depending on the corresponding FMO energy gaps. This dichotomy makes it challenging to correlate reactivity with alkyne electrophilicity; thus, reactivitytrends in SNO-OCT reactions with azides were instead proposed to track with the relevant energy gaps. Switching the alkyl side chain from $\mathrm{R}=\mathrm{Me}$ in 4 to $\mathrm{R}=\mathrm{CH}_{2} \mathrm{OH}$ in 2 was predicted to double the reaction rate with D4. dF-SNOOCT 3 was expected to react even more quickly, with a $k_{2}$ value 

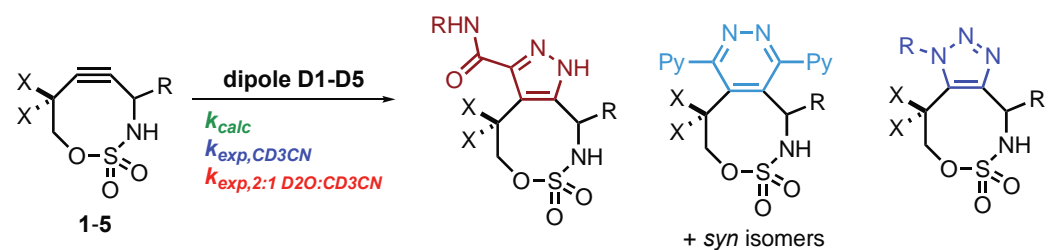

a.

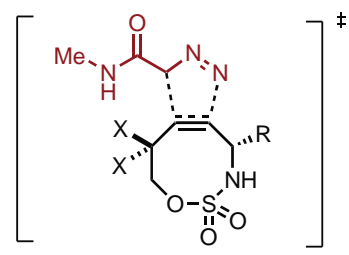

b.

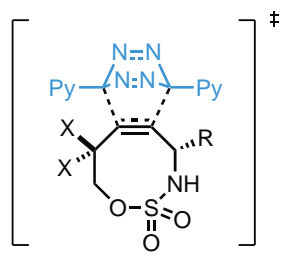

c.

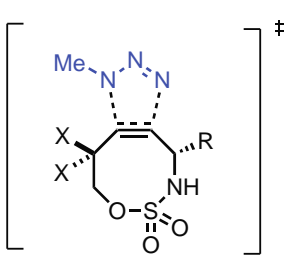

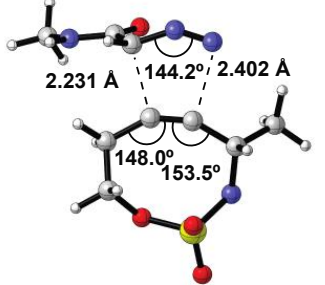
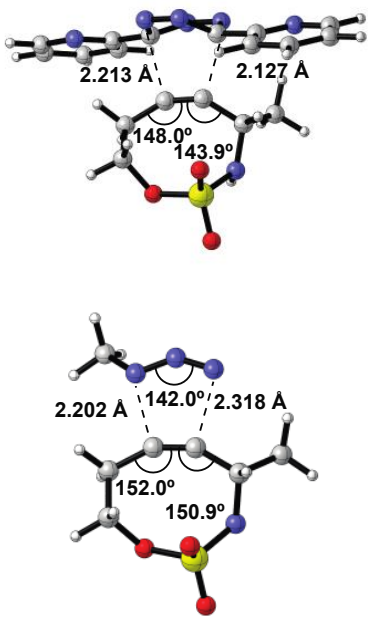
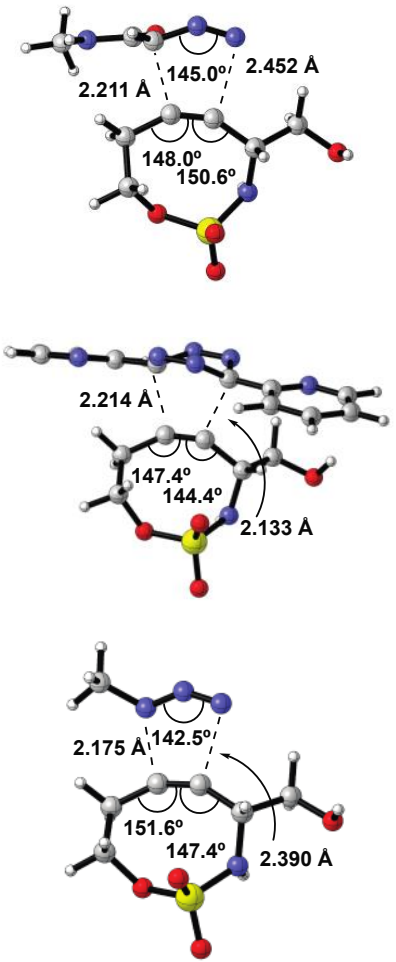
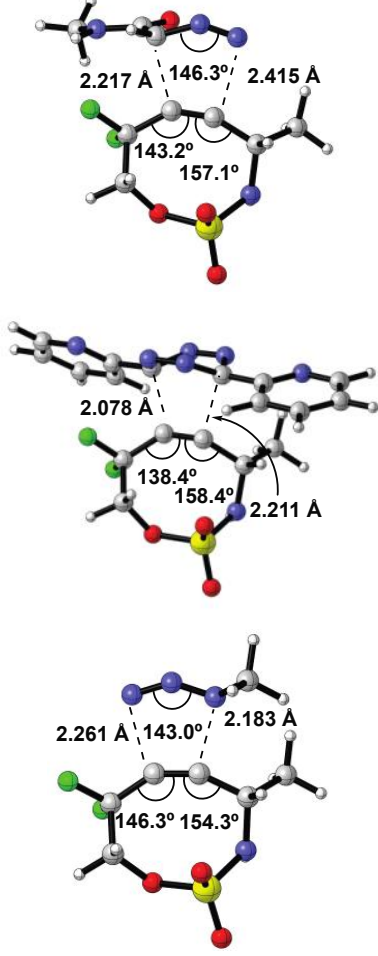

\begin{tabular}{|c|c|c|c|c|c|c|c|c|c|c|c|c|}
\hline \multirow[b]{2}{*}{ SNO-OCT } & \multicolumn{4}{|c|}{ a } & \multicolumn{4}{|c|}{ b } & \multicolumn{4}{|c|}{ c } \\
\hline & $E_{d i s, S N O O C T}$ & $E_{\text {dis,dipole }}$ & $E_{i n t}$ & $E_{\text {act }}$ & $E_{d i s, S N O O C T}$ & $E_{\text {dis,dipole }}$ & $E_{i n t}$ & $E_{\text {act }}$ & $E_{d i s, S N O O C T}$ & $E_{\text {dis,dipole }}$ & $E_{i n t}$ & $E_{\text {act }}$ \\
\hline 4 & 3.46 & 16.2 & 12.7 & 6.96 & 7.14 & 18.9 & 24.8 & 1.17 & 2.53 & 15.2 & 9.48 & 8.21 \\
\hline 2 & 4.15 & 15.5 & 13.2 & 6.49 & 7.49 & 18.5 & 25.7 & 0.27 & 2.61 & 15.1 & 9.87 & 7.81 \\
\hline 3 & 3.55 & 14.4 & 15.8 & 2.21 & 7.99 & 21.3 & 23.4 & 5.86 & 3.46 & 14.4 & 12.0 & 5.93 \\
\hline
\end{tabular}

e.

\begin{tabular}{|c|c|c|c|c|c|c|c|c|c|c|c|c|}
\hline \multirow[b]{2}{*}{ SNO-OCT } & \multicolumn{4}{|c|}{ a } & \multicolumn{4}{|c|}{ b } & \multicolumn{4}{|c|}{ c } \\
\hline & dipole & $\Delta \boldsymbol{G}^{\pi}$ & $k_{\text {calc }}$ & $k_{\text {exp }}$ & dipole & $\Delta \boldsymbol{G}^{\pi}$ & $k_{\text {calc }}$ & $k_{\text {exp }}$ & dipole & $\Delta \mathbf{G}^{\pi}$ & $\boldsymbol{k}_{\text {calc }}$ & $k_{\text {exp }}$ \\
\hline $4 / 1$ & D1/D2 & 19.3 & 0.0475 & 0.270 & D3 & 17.4 & 1.12 & 0.017 & D4/D5 & 20.7 & 0.00395 & 0.026 \\
\hline 2 & & 19.2 & 0.0507 & 0.0582 & & 16.4 & 5.56 & 0.0066 & & 20.0 & 0.0143 & $0.0870(0.130)$ \\
\hline $3 / 5$ & & 15.9 & 13.8 & $>1$ & & 22.7 & 0.00014 & no rxn & & 19.2 & 0.0528 & $0.46(>1)$ \\
\hline
\end{tabular}

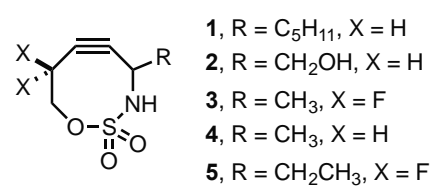

R.

D1, $\mathrm{R}=\mathrm{Me}$

D2, $\mathrm{R}=\mathrm{Bn}$

$$
\overbrace{N^{=}=\mathrm{N}}^{N}
$$

D3
$R^{-N^{*}} N_{i N}^{\oplus} \ominus$

D4, $R=$ Me

D5, $R=B n$

Figure 3. B97D/6-311+G(d,p) calculated transition state models for reactions of SNO-OCTs with (a) diazoacetamide, (b) tetrazine, and (c) azide. (d) Distortion/interaction analysis indicating the influence of distortion energy $\left(\Delta E_{\mathrm{dis}}\right)$ and interaction energy $\left(\Delta E_{\text {int }}\right)$ on activation barriers for cycloadditions of Type I-III dipoles with various SNO-OCT derivatives. (e) Calculated and experimental second-order rate constants. Truncated SNO-OCTs 3 and $\mathbf{4}$ and dipoles D1 and D4 were used for computations; analogous SNO-OCTs 1 and 5 and dipoles D2 and D5 were used for experiments. Energies ( $E_{\text {dis,SNO-OCT, }} E_{\text {dis,dipole }}, E_{\text {int }}, E_{\text {act, }}$ and $\left.\Delta G^{\ddagger}\right)$ are reported in kcal $/ \mathrm{mol}$ and second-order rate constants $\left(k_{\text {calc }}\right.$ and $\left.k_{\text {exp }}\right)$ are in $\mathrm{M}^{-1} \mathrm{~s}^{-1}$. 
predicted to be an order of magnitude higher than with 2 . Indeed, this reactivity trend can be predicted by assessing FMO gaps for each SNO-OCT/azide pair. Higher electron density in $\mathbf{2}$ and $\mathbf{4}$ leads to HOMO destabilization, and as such, these SNOOCTs react via a reduced $\mathrm{HOMO}_{\text {dipolarophile }}-\mathrm{LUMO}_{\text {dipole }}$ gap. Conversely, the presence of the fluoro groups in $\mathrm{dF}-\mathrm{SNO}-\mathrm{OCT}$ 3 increases this gap through HOMO stabilization, thus the $3 / \mathrm{MeN}_{3}$ reaction proceeds through a more favorable $\mathrm{LUMO}_{\text {dipolarophile }}-\mathrm{HOMO}_{\text {dipole }}$ interaction. Though dF-SNOOCT 3 exhibits reduced dipole distortion energy $(-0.72$ $\mathrm{kcal} \cdot \mathrm{mol}^{-1}$ relative to 4$)$, consistent with bond relaxation $\left(+1.0^{\circ}\right)$, the corresponding alkyne distortion energy is elevated in comparison to parent SNO-OCT $4\left(+0.93 \mathrm{kcal} \mathrm{mol}^{-1}\right)$. Thus, the predicted acceleration for cycloadditions of azides with $\mathrm{dF}$ SNO-OCT 3 is attributable to this pair having the highest stabilizing interaction energy $\left(12.0 \mathrm{kcal} \mathrm{mol}^{-1}\right)$. Indeed, the activation barriers show improved correlation with the interaction energies relative to the distortion energies (Figure S1-6).

Experimental Observations Validate Computational Trends. To determine the extent to which SNO-OCT substitution impacts the value of $k_{2}$ in reactions with Type I-III dipoles, the reactivities of $\mathbf{2}-\mathbf{4}$ were investigated experimentally and compared to predicted values. SNO-OCTs $\mathbf{1}$ and $\mathbf{2}$ were prepared as previously reported, ${ }^{9}$ while $\mathbf{5}$ required a modified synthetic route, affording the product in $34 \%$ yield from propionaldehyde (see Scheme S2-1 for details). Computational results were corroborated in selected instances through experimental kinetic data using ${ }^{1} \mathrm{H}$ NMR spectroscopy and plate-reader techniques (Figure 3e). It is important to note that the plate reader was utilized for dipyridyl tetrazine reactions, due to solubility issues encountered in NMR kinetic experiments.

Calculated and experimental results of reactions between SNO-OCTs and alkyl diazoacetamides were particularly promising with respect to chemselectivity and accelerated reaction rates (Figure 3e). Although computed models utilized truncated versions of the substrates, the predicted $k_{2}$ ratio of $\mathbf{4}$ with azide D4 and diazoalkane D1 (12:1) was in excellent agreement with the experimental $k_{2}$ ratio of 1 with D5 and D2 (10:1) (Figure 3e). ${ }^{9}$ A gem-difluorination led to a predicted rate enhancement of $\sim 300$-fold moving from 4 to 3 in reaction with diazoacetamides (D1). Experimental determination of $k_{2}$ for the cycloaddition of $\mathbf{5}$ and $\mathbf{D 2}$ proved difficult; the reaction at rt was too fact to accurately determine $k_{2}$ by NMR. VT-NMR showed formation of 5a with $80 \%$ conversion within 2 min at $-32{ }^{\circ} \mathrm{C}$ (Figure S2-17). Despite the lack of accurate rate data, the fact this reaction is faster than the NMR timescale suggests that $k_{2}>$ $1 \mathrm{M}^{-1} \mathrm{~s}^{-1}$

Predicted reactions of 2-4 with dipyridyltetrazine D3 indicate $~ 8000$-fold rate enhancement in moving from electronpoor dF-SNO-OCT 3 to electron-rich parent 4 ; indeed, experimental observations corroborated this prediction, and no reaction was noted between 5 and D3. Though calculated rates for IEDDA with D3 showed a 2-3 orders of magnitude deviation from experimental rates, the computational method was successful in identifying two promising, mutually orthogonal reactions of SNO-OCTs. Further computational investigation into the origin of this deviation is underway to predict more accurate rates in IEDDA with $\mathbf{D 3}$ for future studies.
Calculated rates between azides and SNO-OCTs fall within one order of magnitude from experimental values, when conducting measurements in $\mathrm{CD}_{3} \mathrm{CN}$ (Figure $3 \mathrm{e}$ ). ${ }^{9}$ The predicted value of $k_{\text {rel,azide }}$ between 2:4 (3.6:1) was in excellent agreement with the experimental value of $k_{\text {rel,azide }}$ between 2:1 (3.3:1). Furthermore, calculations indicated a $13 \times$ rateenhancement moving from parent $\mathbf{4}$ to dF-SNO-OCT 3, compared to the experimentally observed $18 \times$ rateenhancement in moving from $\mathbf{1}$ to $\mathbf{5}$, accurately predicting relevant experimental reactivity trends. Modified SNO-OCTs 4 and 1 also show predictable trends for azide/SNO-OCT cycloadditions. To our surprise, the computed $k_{\text {rel,azide }}$ between 3:2 (3.7:1) showed a better agreement with experimental results for 5:2 (3.5:1) when more water was used $\left(2: 1 \mathrm{D}_{2} \mathrm{O}: \mathrm{CD}_{3} \mathrm{CN}\right)$, suggesting a potential hydrophobic effect contributing to rate acceleration. ${ }^{22}$

The inability of DFT methods to accurately model solvents is well-known. Zuilhof and coworkers also point out that their solvent model does not account for rate increases dependent on solvent identity. ${ }^{15}$ In our case, the B97D functional is sufficient to predict how electronic fine-tuning of the SNO-OCT influences cycloaddition rates, particularly in [3+2] cycloadditions.

Development of Dual and Triple Ligation Systems with Custom SNO-OCTs. With initial experimental and computational data in hand, we wanted to develop mutually exclusive bioorthogonal dual or triple ligations of SNO-OCTs with various dipoles, as dual ligation has been shown to be effective in probing complex native biological environments and details of subcellular mechanisms. ${ }^{23}$ Success would effectively expand the tool box available for multiple ligation of biologically relevant functionalities and also enable finetuning of the properties of the SNO-OCT for specific applications.

As predicted by DFT, SNO-OCT 5 gave excellent yields in reactions with diazoalkane D2 $(94 \%)$ and azide D5 (96\%); no reaction was noted with $\mathbf{D 3}$ (Scheme 1a). In validation of our initial hypothesis that a dual ligation system comprised of orthogonal SNO-OCTs and Type I/Type III dipoles could be achieved, a competition experiment using SNO-OCTs $\mathbf{1}$ and $\mathbf{5}$ and dipoles D2 and D3 (Schemes 1b, S2-2a, and S2-2b; Figure S2-23) showed complete mutual orthogonality. ${ }^{2 \mathrm{~g}, 4 \mathrm{~d}, 24}$ Indeed, the excellent chemoselectivity supported the large rate enhancement predicted in moving from parent 4 to $\mathrm{dF}-\mathrm{SNO}$ OCT 3. In contrast, a dual ligation system with azide $\mathbf{D 5}$ instead of $\mathbf{D 3}$ gave significantly reduced chemoselectivity (Scheme S22c); this also matches computed reaction rates (Figure $3 \mathrm{e}$ ).

The development of a triple ligation system using only custom SNO-OCTs proved challenging, as predicted by the computational rate data. Ideally, the three mutually exclusive pairs of reagents should display $k_{2}$ values differing by at least two orders of magnitude. Based on calculated relative rates of dF-SNO-OCT 3/D1 and parent 4/D1 (Figure 3e), this condition cannot be explicitly met with our current SNO-OCT derivatives. $^{14}$ Thus, a commercially available non-SPAAC boronic acid/hydrazine pair was introduced. Bane and coworkers have shown that boronic acid and hydrazine/hydrazide pairs are competent in fast bioorthogonal labeling of biologically relevant molecules; furthermore, the boronic acid system shows orthogonality to both IEDDA and SPAAC. ${ }^{2 \mathrm{~h}, 25}$ The orthogonality of these reactions was verified by LC-MS analysis, which is more reliable than NMR methods when 
significant peak overlap occurs in complex mixtures. ${ }^{2 g}$ To our delight, the orthogonality in Scheme $1 \mathrm{~b}$ translated to the triple ligation (Scheme 1c, Figure S2-17), with mutual orthogonality observed amongst all three reaction pairs by LC-MS.

Protein Triple Ligation and Mammalian Cell-Labeling Assays. Simultaneous multitarget in vitro and in vivo labeling is of considerable interest for advancing modern biotherapeutics, biological assay development, and basic science investigations. ${ }^{26} \mathrm{We}$ sought to demonstrate the mutual orthogonality of our SNO-OCT bioorthogonal pairs in biological settings, as well as their capacity to enable tripleorthogonal ligations as suggested by LC-MS assays (Scheme 1c). To that end, SNO-DF-Rho and SNO-C343 were synthesized from $\mathbf{5}$ and $\mathbf{1}$, employing either carboxylic acid or primary amine handles, as they are easily derivatizable with other biologically relevant functionalities (Scheme S2-3). Additionally, a previously reported cysteine-hydrazide Texas Red derivative ${ }^{2 \mathrm{~h}}$ was also adapted (Figure $4 \mathrm{a}$, see the SI for more details). Based on the LC-MS experiment in Scheme 1c, these reagents should form the basis of a tripartite mutually orthogonal set, appropriate for demonstrating translatability to biologically relevant conditions. Indeed, one-pot labeling of three different proteins functionalized to present a diazoacetamide (Cytoc-AcDz), tetrazine (RNase A-Tez), and boronic acid-aldehyde (RNase A-BA) showed excellent chemoselectivity in agreement with small-molecule triple ligation results (Figure 4a). As anticipated, in situ gel fluorescence images revealed some bleed over between dyes, but minimal cross reactivity across orthogonal pairs, demonstrating the chemoselectivity of the reagents (Figure S218).

Next, we sought to explore potential applications in cellulo. The cellular uptake properties of SNO-C343, SNO-DF-Rho, and three DBCO-derivatives were compared to assess internalization and localization of these molecules within Chinese hamster ovary ( $\mathrm{CHO})-\mathrm{K} 1$ cells. We observed the rapid internalization of SNO-C343 and SNO-DF-Rho (Figures 4b, S2-19, and S2-20). Colocalization analysis of SNO-DF-Rho with MitoTracker Green ${ }^{\mathrm{TM}}$ revealed robust mitochondrial trafficking as shown by a Pearson's correlation coefficient of $R$ $=0.90 \pm 0.01$ (Figure S2-21). Comparison to an in-house synthesized DBCO-C343 derivative as well as two commercial conjugates, DBCO-PEG $4-\mathrm{Rh}_{4} 10$ and DBCO-Cy5, revealed enhanced internalization of SNO-OCT-dye conjugates relative to the commercial reagents upon incubation with $\mathrm{CHO}-\mathrm{K} 1$ cells (Figure 4b). Internalization of DBCO-C343 occurred at comparable concentrations and gave staining patterns similar to those observed for SNO-C343 (Figure 4B, Figure S2-23). Internalization experiments with $\mathrm{DBCO}-\mathrm{PEG}_{4}-\mathrm{Rhol}_{10}$ and DBCO-Cy5 conjugates illustrated indirect internalization of DBCO-PEG 4 -Rho110, and ablation of internalization in the case of DBCO-Cy5, even at concentrations 5-fold greater than the maximum explored with SNO-OCT reagents (Figure $4 \mathrm{~b}$ and Figure S2-22). In addition to the orthogonality of SNO-DFRho and SNO-C343 reactivity with Type-I and Type-II dipoles, these unconjugated reagents are taken-up robustly by mammalian cells and thus have potential for in cellulo applications.

The mammalian glycocalyx forms a protective anionic coating anchored to the lipid bilayer. ${ }^{27}$ The structure and chemical properties of this bilayer play important roles in both normal and pathological biological processes, including cellular recognition, organismal development, cancer, and infectivity. ${ }^{28}$ In pioneering investigations, Reutter, Bertozzi, and their coworkers labeled the glycocalyx of live cells metabolically with synthetic carbohydrates. ${ }^{29}$ We sought to enlarge the toolkit for glycocalyx analyses with the new SNO-OCT reagents.

We used metabolic labelling with $\mathrm{Ac}_{4} \mathrm{ManDiaz}^{22}$ to assess the reactivity of SNO-DF-Rho in a biological context. We began by assessing the concentration-dependent uptake and distribution of $\mathrm{Ac}_{4} \mathrm{ManDiaz}$ in $\mathrm{CHO}-\mathrm{K} 1$ cells, identifying optimal concentrations for resolving the glycocalyx upon reaction with SNO-DF-Rho at $1 \mu \mathrm{M}$ (Figure S2-24). Side-byside comparison of staining patterns in $\mathrm{CHO}-\mathrm{K} 1$ cells cultured in the absence or presence of $\mathrm{Ac}_{4} \mathrm{ManDiaz}$ revealed a difference in the distribution of the label. Specifically, SNO-DF-Rho when cells were metabolically labeled with $\mathrm{Ac}_{4} \mathrm{ManDiaz}$ (Figure $4 \mathrm{~d}$ and $4 \mathrm{e}$ ). In analogous flow-cytometry experiments, the robust labeling and internalization of cells metabolically

Scheme 1. Successful mutually orthogonal ligation systems using SNO-OCTs and Type I/III dipoles. (a) SNO-OCT 5 shows distinct chemoselectivity for D2 and D5 over D3. (b) Successful development of a dual SNO-OCT ligation system. (c) Triple ligation system assessed by LC-MS analysis (at $210 \mathrm{~nm}$ ). *By-product from cycloaddition between $\mathbf{4}$ and D3. $* * \mathbf{5}$ and D2 product observed at high sample concentration (see the SI for more information).
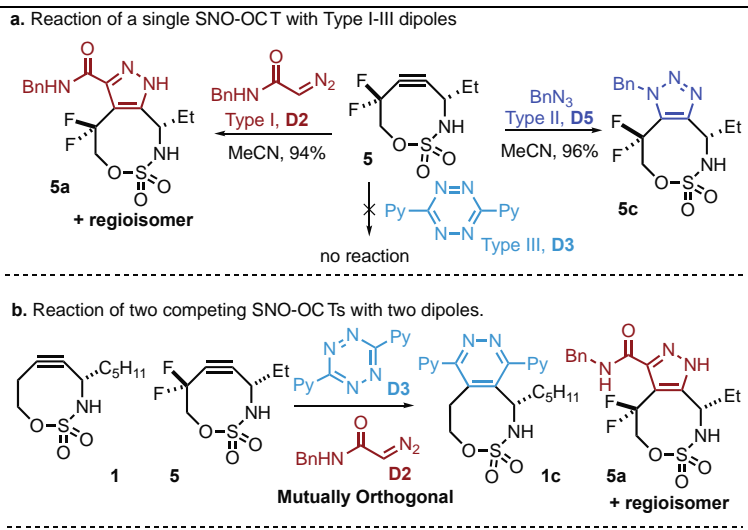

c. Reaction of three mutually orthogonal pairs.
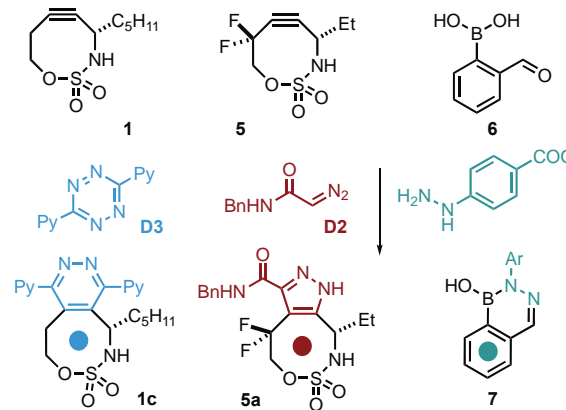

5
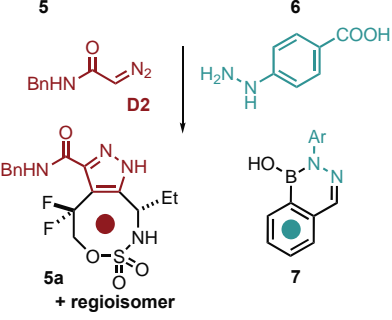

Mutually Orthogonal

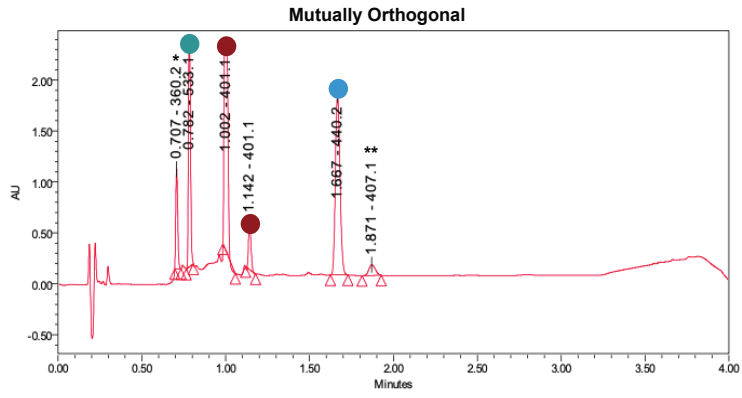


a
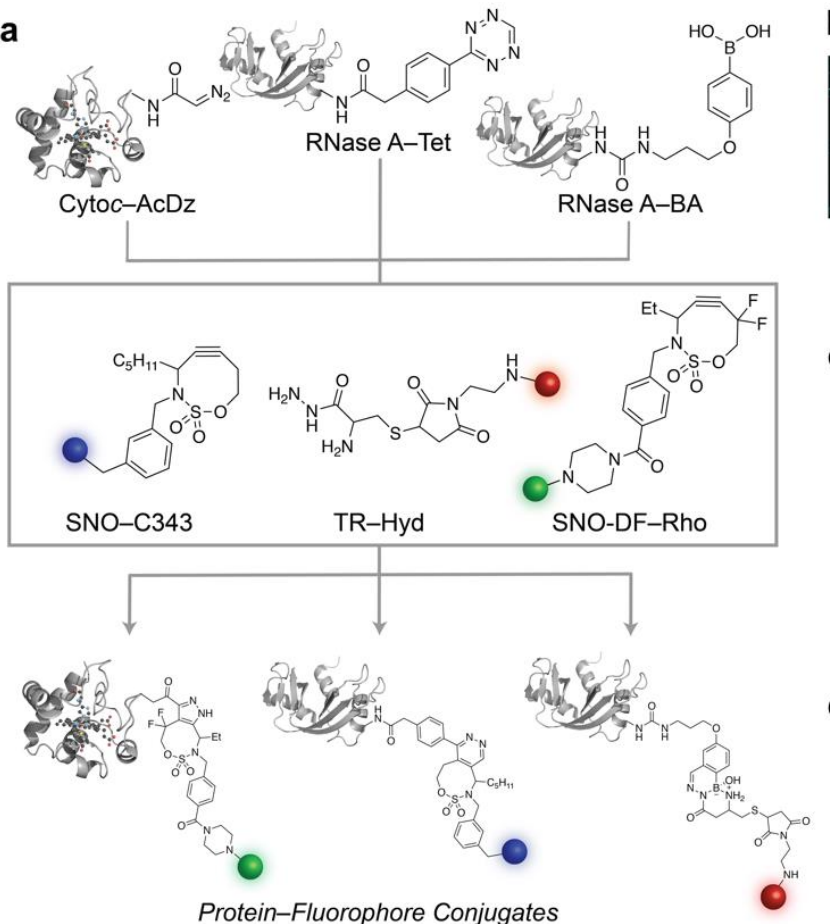

Protein-Fluorophore Conjugates b

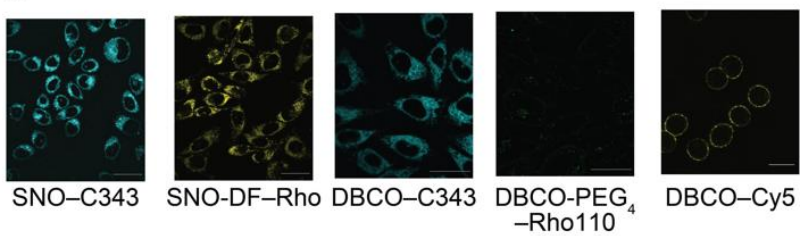

C
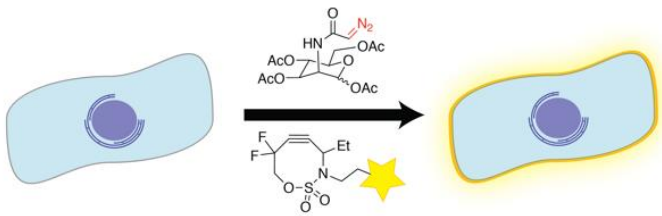

d

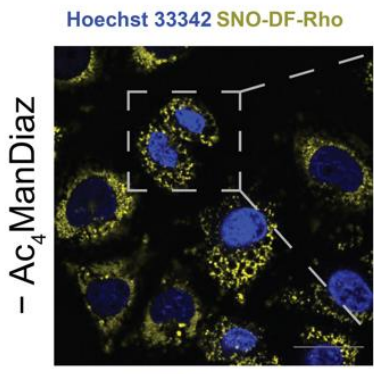

Hoechst 33342 SNO-DF-Rho
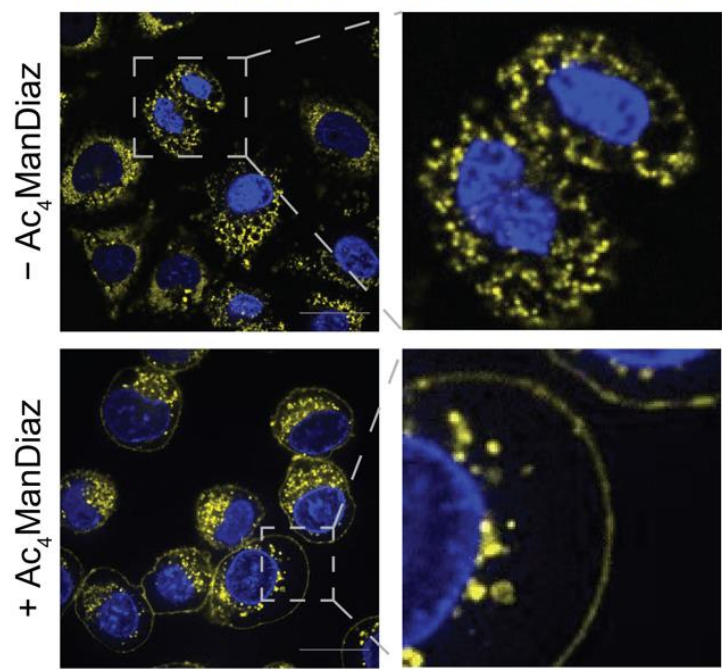

Figure 4. Triple ligation and in cellulo activity of SNO-OCT-dye conjugates. (a) Co-incubation of proteins that were labeled with reactive functional groups and fluorescent reagents. (b) Internalization of cyclooctyne-dye conjugates $(1 \mu \mathrm{M})$ into CHO-K1 cells. Scale: $10 \mu \mathrm{m}$. (c) Scheme of the metabolic labeling experiments. (d) Visualization of metabolically labeled CHO-K1 cells grown with (bottom) or without (top) Ac 4 ManDiaz (20 $\mu \mathrm{M})$ then incubated with SNO-DF-Rho $(1 \mu \mathrm{M})$ for $1 \mathrm{~h}$. Scale: $10 \mu \mathrm{m}$.

labeled with $\mathrm{Ac}_{4} \mathrm{ManDiaz}$ was again observed, and without significant cell death (Figure S2-25). Together, these data demonstrate the reactivity of SNO-DF-Rho in cellulo and highlight the potential of SNO-OCT reagents for biological assays in living cells.

\section{CONCLUSIONS}

The integration of computational and experimental studies led to custom heterocyclic cycloalkynes (SNO-OCTs) that display orthogonal reactivities with Type I and III dipoles. The cooperative effects of the propargylic and homopropargylic heteroatoms, which are separated by a sulfonyl bridge, enable tuning of the alkyne electronics and alkyne distortion via remote hybridization and stereoelectronic effects. The addition of a gem-difluoro group to the propargylic carbon of the SNO-OCT has several beneficial features, including a significant increase in the value of $k_{2}$ for reactions with Type I-II dipoles and a greatly decreased preference for participation in IEDDA-type reactions. Inspired by computational investigations, experimental validation of a new mutually orthogonal dual ligation was realized through successful electronic fine-tuning of the SNO-OCT alkyne. The inclusion of a previously reported boronic acid/hydrazine bioorthogonal pair furnished a triple ligation system demonstrated to be mutually orthogonal. The development of highly chemoselective, bioorthogonal labeling reactions for double and triple ligations provides useful new tools for future in cellulo investigations. The utility of SNOOCTs for biological applications was demonstrated through a series of protein and cellular labeling assays. The full synthetic potential of these strained alkynes is an area of ongoing investigation in our research groups, with the goal of creating easily accessible and robust bioorthogonal reagents to accelerate biological discovery.

\section{ASSOCIATED CONTENT}

Characterization data, optimization tables, and additional substrates/catalysts are included in the supplementary materials, which are available free of charge via the Internet at http://pubs.acs.org.

\section{AUTHOR INFORMATION}

*schomakerj@chem.wisc.edu 
*rtraines@mit.edu

\section{Author Contributions}

The manuscript was written by J.M.S., R.T.R., J.M.R., Y.H., and H.R.K. Experimental work was conducted by Y.H, H.R.K., and A.S.M.L. Computational work was conducted by J.M.R. Biological work was conducted by H.R.K. All authors have given approval to the final version of the manuscript.

\section{Funding Sources}

J.M.S. is grateful to the Wisconsin Alumni Research Foundation for financial support of this research. H.R.K. and R.T.R. were supported by grant NIH R01 GM044783. The Paul Bender Chemistry Instrumentation Center includes: Thermo Q ExactiveTM Plus by NIH 1S10 OD020022-1; Bruker Avance-500 by a generous gift from Paul J. and Margaret M. Bender; Bruker Avance-600 by NIH S10 OK012245; Bruker Avance-400 by NSF CHE-1048642; Bruker D8 VENTURE Photon III by NSF CHE1919350 and the University of Wisconsin-Madison; Varian Mercury-300 by NSF CHE-0342998.

\section{ACKNOWLEDGMENT}

Dr. Charles G. Fry and Dr. Heike Hofstetter at UW-Madison are thanked for help with NMR techniques. Dr. Martha M. Vestling at UW-Madison is thanked for mass spectrometry characterization.

\section{REFERENCES}

(1) For reviews: (a) Agard, N. J.; Prescher, J. A.; Bertozzi, C. R. A Strain-Promoted [3 + 2] Azide-Alkyne Cycloaddition for Covalent Modification of Biomolecules in Living Systems. J. Am. Chem. Soc. 2004, 126, 15046-15047. (b) Sletten, E. M.; Bertozzi, C. R. Bioorthogonal Chemistry: Fishing for Selectivity in a Sea of Functionality. Angew. Chem., Int. Ed. 2009, 48, 6974-6998. (c) Sletten, E. M.; Bertozzi, C. R. From Mechanism to Mouse: A Tale of Two Bioorthogonal Reactions. Acc. Chem. Res. 2011, 44, 666-676. (d) Ngo, J. T.; Tirrell, D. A. Noncanonical Amino Acids in the Interrogation of Cellular Protein Synthesis. Acc. Chem. Res. 2011, 44, 677-685. (e) Willems, L. I.; Van Der Linden, W. A.; Li, N.; Li, K. Y.; Liu, N.; Hoogendoorn, S.; Van Der Marel, G. A.; Florea, B. I.; Overkleeft, H. S. Bioorthogonal Chemistry: Applications in Activity-Based Protein Profiling. Acc. Chem. Res. 2011, 44, 718-729. (f) Best, M. D.; Rowland, M. M.; Bostic, H. E. Exploiting Bioorthogonal Chemistry to Elucidate Protein-Lipid Binding Interactions and Other Biological Roles of Phospholipids. Acc. Chem. Res. 2011, 44, 686-698. (g) Hang, H. C.; Wilson, J. P.; Charron, G. Bioorthogonal Chemical Reporters for Analyzing Protein Lipidation and Lipid Trafficking. Acc. Chem. Res. 2011, 44, 699-708. (h) Hao, Z.; Hong, S.; Chen, X.; Chen, P. R. Introducing Bioorthogonal Functionalities into Proteins in Living Cells. Acc. Chem. Res. 2011, 44, 742-751. (i) Chen, Y. X.; Triola, G.; Waldmann, H. Bioorthogonal Chemistry for Site-Specific Labeling and Surface Immobilization of Proteins. Acc. Chem. Res. 2011, 44, 762773. (j) Jing, C.; Cornish, V. W. Chemical Tags for Labeling Proteins inside Living Cells. Acc. Chem. Res. 2011, 44, 784-792. (k) Debets, M. F.; Van Berkel, S. S.; Dommerholt, J.; Dirks, A. J.; Rutjes, F. P. J. T.; Van Delft, F. L. Bioconjugation with Strained Alkenes and Alkynes. Acc. Chem. Res. 2011, 44, 805-815. (1) Devaraj, N. K.; Weissleder, R. Biomedical Applications of Tetrazine Cycloadditions. Acc. Chem. Res. 2011, 44, 816-827. (m) Lang, K.; Chin, J. W. Bioorthogonal Reactions for Labeling Proteins. ACS Chem. Biol. 2014, 9, 16-20. (n) Oliveira, B. L.; Guo, Z.; Bernardes, G. J. L. Inverse Electron Demand Diels-Alder Reactions in Chemical Biology. Chem. Soc. Rev. 2017, 46, 4895-4950.

(2) For reviews: (a) Liang, Y.; Mackey, J. L.; Lopez, S. A.; Liu, F.; Houk, K. N. Control and Design of Mutual Orthogonality in Bioorthogonal Cycloadditions. J. Am. Chem. Soc 2012, 134, 1790417907. (b) Patterson, D. M.; Nazarova, L. A.; Prescher, J. A. Finding the Right (Bioorthogonal) Chemistry. ACS Chem. Biol. 2014, 9, 592 605. (c) Patterson, D. M.; Prescher, J. A. Orthogonal Bioorthogonal
Chemistries. Curr. Opin. Chem. Biol. 2015, 28, 141-149. (d) David Row, R.; Prescher, J. A. Constructing New Bioorthogonal Reagents and Reactions. Acc. Chem. Res. 2018, 51, 1073-1081. (e) Devaraj, N. K. The Future of Bioorthogonal Chemistry. ACS Cent. Sci. 2018, 4, 952-959. Recent publications: (f) Karver, M. R.; Weissleder, R.; Hilderbrand, S. A. Bioorthogonal Reaction Pairs Enable Simultaneous, Selective, Multi-Target Imaging. Angew. Chem., Int. Ed. 2012, 51, 920-922. (g) Kamber, D. N.; Nguyen, S. S.; Liu, F.; Briggs, J. S.; Shih, H. W.; Row, R. D.; Long, Z. G.; Houk, K. N.; Liang, Y.; Prescher, J. A. Isomeric Triazines Exhibit Unique Profiles of Bioorthogonal Reactivity. Chem. Sci. 2019, 10, 9109-9114. (h) Chio, T. I.; Gu, H.; Mukherjee, K.; Tumey, N.; Bane, S. L. Site-Specific Bioconjugation and Multi-Bioorthogonal Labeling via Rapid Formation of BoronNitrogen Heterocycle. Bioconjugate Chem. 2019, 30, 1554-1564. (i) Schäfer, R. J. B.; Monaco, M. R.; Li, M.; Tirla, A.; Rivera-Fuentes, P.; Wennemers, H. The Bioorthogonal Isonitrile-Chlorooxime Ligation. J. Am. Chem. Soc 2019, 141, 18644-18648.

(3) (a) Chen, W.; Wang, D.; Dai, C.; Hamelberg, D.; Wang, B. Clicking 1,2,4,5-Tetrazine and Cyclooctynes with Tunable Reaction Rates. Chem. Commun. 2012, 48, 1736-1738. (b) MacKenzie, D. A.; Sherratt, A. R.; Chigrinova, M.; Cheung, L. L. W.; Pezacki, J. P. StrainPromoted Cycloadditions Involving Nitrones and Alkynes-Rapid Tunable Reactions for Bioorthogonal Labeling. Curr. Opin. Chem. Biol. 2014, 21, 81-88. (c) Kamber, D. N.; Nazarova, L. A.; Liang, Y.; Lopez, S. A.; Patterson, D. M.; Shih, H.-W.; Houk, K. N.; Prescher, J. A. Isomeric Cyclopropenes Exhibit Unique Bioorthogonal Reactivities. J. Am. Chem. Soc 2013, 135, 13680.

(4) For reviews: (a) Debets, M. F.; Van Berkel, S. S.; Dommerholt, J.; Dirks, J.; Rutjes, F. P. J. T.; Van Delft, F. L. Bioconjugation with Strained Alkenes and Alkynes Bioconjugation with Strained Alkenes and Alkynes. Acc. Chem. Res. 2011, 44, 805-815. (b) Huber, T.; Sakmar, T. P. Chemical Biology Methods for Investigating G ProteinCoupled Receptor Signaling. Chem. Biol. 2014, 21, 1224-1237. (c) Dommerholt, J.; Rutjes, F. P. J. T.; Van Delft, F. L. Strain-Promoted 1,3-Dipolar Cycloaddition of Cycloalkynes and Organic Azides. Top Curr. Chem. 2016, 374, 16-36. Recent publications: (d) Reddington, S. C.; Tippmann, E. M.; Dafydd Jones, D. Residue Choice Defines Efficiency and Influence of Bioorthogonal Protein Modification via Genetically Encoded Strain Promoted Click Chemistryw. Chem. Commun. 2012, 48, 8419-8421. (e) Aronoff, M. R.; Gold, B.; Raines, R. T. 1,3-Dipolar Cycloadditions of Diazo Compounds in the Presence of Azides. Org. Lett. 2016, 18, 1538-1541. f) Kaneda, K.; Naruse, R.; Yamamoto, S. 2-Aminobenzenesulfonamide-Containing Cyclononyne as Adjustable Click Reagent for Strain-Promoted Azide-Alkyne Cycloaddition. Org. Lett. 2017, 19, 1096-1099.

(5) Baskin, J. M.; Prescher, J. A.; Laughlin, S. T.; Agard, N. J.; Chang, P. V.; Miller, I. A.; Lo, A.; Codelli, J. A.; Bertozzi, C. R. Copper-Free Click Chemistry for Dynamic in Vivo Imaging. Proc. Natl. Acad. Sci. USA 2007, 104, 16793-16797.

(6) (a) Golkowski, M.; Ziegler, T. Synthesis of Tetra(2Hydroxyethoxy)-Substituted Dibenzocyclooctyne Derivatives as Novel, Highly Hydrophilic Tool Compounds for Strain-Promoted Alkyne-Azide Cycloaddition Applications. Synthesis. 2013, 45, 1207 1214. (b) Debets, M. F.; Prins, J. S.; Merkx, D.; Van Berkel, S. S.; Van Delft, F. L.; Van Hest, J. C. M.; Rutjes, F. P. J. T. Synthesis of DIBAC Analogues with Excellent SPAAC Rate Constants. Org. Biomol. Chem. 2014, 12, 5031-5037. (c) Chadwick, R. C.; Van Gyzen, S.; Liogier, S.; Adronov, A. Scalable Synthesis of Strained Cyclooctyne Derivatives. Synthesis. 2014, 46, 669-677.

(7) Debets, M. F.; Van Berkel, S. S.; Schoffelen, S.; Rutjes, F. P. J. T.; Van Hest, J. C. M.; Van Delft, F. L. Aza-Dibenzocyclooctynes for Fast and Efficient Enzyme PEGylation via Copper-Free [3+2] Cycloaddition. Chem. Commun. 2010, 46, 97-99.

(8) (a) McKay, C. S.; Finn, M. G. Click Chemistry in Complex Mixtures: Bioorthogonal Bioconjugation. Chem. Biol. 2014, 21, 10751101. (b) Bakkum, T.; Van Leeuwen, T.; Sarris, A. J. C.; Van Elsland, D. M.; Poulcharidis, D.; Overkleeft, H. S.; Van Kasteren, S. I. Quantification of Bioorthogonal Stability in Immune Phagocytes Using Flow Cytometry Reveals Rapid Degradation of Strained Alkynes. ACS 
Chem. Biol. 2018, 13, 1173-1179.

(9) Burke, E. G.; Gold, B.; Hoang, T. T.; Raines, R. T.; Schomaker, J. M. Fine-Tuning Strain and Electronic Activation of Strain-Promoted 1,3-Dipolar Cycloadditions with Endocyclic Sulfamates in SNOOCTs. J. Am. Chem. Soc 2017, 139, 8029-8037.

(10) Tu, J.; Svatunek, D.; Parvez, S.; Liu, A. C.; Levandowski, B. J.; Eckvahl, H. J.; Peterson, R. T.; Houk, K. N.; Franzini, R. M. Stable, Reactive and Orthogonal Tetrazines: Dispersion Forces Promote the Cycloaddition with Isonitriles. Angew. Chem., Int. Ed. 2019, 58, 9043 9048.

(11) Glendening, E. D.; Badenhoop, J. K.; Reed, A. E.; Carpenter, J. E.; Bohmann, J. A.; Morales, C. M.; Landis, C. R.; Weinhold, F. NBO 6.0, Theoretical Chemistry Institute, University of Wisconsin, Madison, 2013

(12) (a) Hohenstein, E. G.; Chill, S. T.; Sherrill, C. D. Assessment of the Performance of the M05-2X and M06-2X Exchange-Correlation Functionals for Noncovalent Interactions in Biomolecules. J. Chem. Theory Comput 2008, 4, 1996-2000. (b) Walker, M.; Harvey, A. J. A.; Sen, A.; Dessent, C. E. H. Performance of M06, M06-2X, and M06HF Density Functionals for Conformationally Flexible Anionic Clusters: M06 Functionals Perform Better than B3LYP for a Model System with Dispersion and Ionic Hydrogen-Bonding Interactions. $J$. Phys. Chem. A 2013, 117, 12590-12600.

(13) Frisch, M. J.; Trucks, G. W.; Schlegel, H. B.; Scuseria, G. E.; Robb, M. A.; Cheeseman, J. R.; Scalmani, G.; Barone, V.; Petersson, G. A.; Nakatsuji, H.; Li, X.; Caricato, M.; Marenich, A. V.; Bloino, J.; Janesko, B. G.; Gomperts, R.; Mennucci, B.; Hratch, D. J. Gaussian 16, Revision B.01. Gaussian, Inc.: Wallingford, CT 2016

(14) Liu, F.; Liang, Y.; Houk, K. Bioorthogonal Cycloadditions: Computational Analysis with the Distortion/Interaction Model and Predictions of Reactivities. Acc. Chem. Res. 2017, 50, 2297-2308.

(15) (a) Garcia-Hartjes, J.; Dommerholt, J.; Wennekes, T.; Van Delft, F. L.; Zuilhof, H. Electronic Effects versus Distortion Energies during Strain-Promoted Alkyne-Azide Cycloadditions: A Theoretical Tool to Predict Reaction Kinetics. European J. Org. Chem. 2013, 6 , 3712-3720. (b) Escorihuela, J.; Das, A.; Looijen, W. J. E.; Van Delft, F. L.; Aquino, A. J. A.; Lischka, H.; Zuilhof, H. Kinetics of the StrainPromoted Oxidation-Controlled Cycloalkyne-1,2-Quinone Cycloaddition: Experimental and Theoretical Studies. J. Org. Chem. 2018, 83, 244-252.

(16) (a) Barone, V.; Cossi, M. Quantum Calculation of Molecular Energies and Energy Gradients in Solution by a Conductor Solvent Model. J. Phys. Chem. A 1998, 102, 1995-2001. (b) Cossi, M.; Rega, N.; Scalmani, G.; Barone, V. Energies, Structures, and Electronic Properties of Molecules in Solution with the C-PCM Solvation Model. J. Comput. Chem. 2003, 24, 669-681. (c) Takano, Y.; Houk, K. N. Benchmarking the Conductor-like Polarizable Continuum Model (CPCM) for Aqueous Solvation Free Energies of Neutral and Ionic Organic Molecules. J. Chem. Theory Comput. 2005, 1, 70-77. (d) Grimme, S. Semiempirical GGA-Type Density Functional Constructed with a Long-Range Dispersion Correction. J. Comput. Chem. 2006, 27, 1787-1799. (e) Grimme, S.; Ehrlick, S.; Goerigk, L. Effect of the Damping Function in Dispersion Corrected Density Functional Theory. J. Comput. Chem. 2011, 32, 1456-1465.

(17) Fleming, I. Molecular Orbitals and Organic Chemistry Reactions: Student Edition; Wiley: West Sussex, UK, 2009.

(18) (a) Ess, D. H.; Houk, K. N. Distortion/Interaction Energy Control of 1,3-Dipolar Cycloaddition Reactivity. J. Am. Chem. Soc. 2007, 129, 10646-10647. (b) Ess, D. H.; Houk, K. N. Theory of 1,3Dipolar Cycloadditions: Distortion/Interaction and Frontier Molecular Orbital Models. J. Am. Chem. Soc. 2008, 130, 10187-10198.

(19) (a) Fernández, I.; Bickelhaupt, F. M. The Activation Strain Model and Molecular Orbital Theory: Understanding and Designing Chemical Reactions. Chem. Soc. Rev. 2014, 43, 4953-4967. (b) Wolters, L. P.; Bickelhaupt, F. M. The Activation Strain Model and Molecular Orbital Theory. Wiley Interdiscip. Rev. Comput. Mol. Sci. 2015, 5, 324-343.
(20) Liu, F.; Liang, Y.; Houk, K. N. Theoretical Elucidation of the Origins of Substituent and Strain Effects on the Rates of Diels-Alder Reactions of 1,2,4,5-Tetrazines. J. Am. Chem. Soc. 2014, 136, 11483 11493.

(21) Sadasivam, D. V.; Prasad, E.; Flowers, R. A.; Birney, D. M. Stopped-Flow Kinetics of Tetrazine Cycloadditions; Experimental and Computational Studies toward Sequential Transition States. J. Phys. Chem. A 2006, 110, 1288-1294.

(22) Andersen, K. A.; Aronoff, M. R.; Mcgrath, N. A.; Raines, R. T. Diazo Groups Endure Metabolism and Enable Chemoselectivity in Cellulo. J. Am. Chem. Soc. 2015, 137, 2412-2415

(23) (a) Huang, L. L.; Liu, K.; Zhang, Q.; Xu, J.; Zhao, D.; Zhu, H.; Xie, H. Y. Integrating Two Efficient and Specific Bioorthogonal Ligation Reactions with Natural Metabolic Incorporation in One Cell for Virus Dual Labeling. Anal. Chem. 2017, 89, 11620-11627. (b) Das, D. K.; Govindan, R.; Nikić-Spiegel, I.; Krammer, F.; Lemke, E. A.; Munro, J. B. Direct Visualization of the Conformational Dynamics of Single Influenza Hemagglutinin Trimers. Cell 2018, 174, 926-937.e12. (c) Reisacher, U.; Ploschik, D.; Rönicke, F.; Cserép, G. B.; Kele, P.; Wagenknecht, H.-A. Copper-Free Dual Labeling of DNA by Triazines and Cyclopropenes as Minimal Orthogonal and Bioorthogonal Functions. Chem. Sci. 2019, 10, 4032-4037.

(24) Dommerholt, J.; Van Rooijen, O.; Borrmann, A.; Fonseca Guerra, C.; Bickelhaupt, F. M.; Van Delft, F. L. Highly Accelerated Inverse Electron-Demand Cycloaddition of Electron-Deficient Azides with Aliphatic Cyclooctynes. Nat. Commun. 2014, 5, 5378.

(25) (a) Dilek, O.; Lei, Z.; Bane, S. Rapid Formation of a Stable Boron-Nitrogen Heterocycle in Dilute, Neutral Aqueous Solution for Bioorthogonal Coupling Reactions. Chem. Commun. 2015, 16992 16995. (b) Gu, H.; Ghosh, S.; Staples, R. J.; Bane, S. L. $\beta$-HydroxyStabilized Boron-Nitrogen Heterocycles Enable Rapid and Efficient C-Terminal Protein Modification. Bioconjugate Chem. 2019, 30, 2604-2613. For reviews: (c) Akgun, B.; Hall, D. G. Boronic Acids as Bioorthogonal Probes for Site-Selective Labeling of Proteins. Angew. Chem, Int. Ed. 2018, 57, 13028-13044.

(26) (a) Kolb, H. C.; Finn, M. G.; Sharpless, B. K. Click Chemistry: Diverse Chemical Function from a Few Good Reactions. Angew. Chem., Int. Ed. 2001, 40, 2004-2021. (b) Cravatt, B. F.; Wright, A. T.; Kozarich, J. W. Activity-Based Protein Profiling: From Enzyme Chemistry to Proteomic Chemistry. Annu. Rev. Biochem. 2008, 77, 383-414. (c) Laughlin, S. T.; Bertozzi, C. R. Imaging the Glycome. Proc. Natl. Acad. Sci. U.S.A. 2009, 106, 12-17. (d) Signore, G.; Nifosi, R.; Albertazzi, L.; Storti, B.; Bizzarri, R. Polarity-Sensitive Coumarins Tailored to Live Cell Imaging. J. Am. Chem. Soc. 2010, 132, 12761288. (e) Dennler, P.; Fischer, E.; Schibli, R. Antibody Conjugates: From Heterogenous Populations to Defined Reagents. Antibodies 2015, 4, 197-224. (f) Beck, A.; Goetsch, L.; Dumontet, C.; Corvaïa, N. Strategies and Challenges for the next Generation of Antibody-Drug Conjugates. Nat. Rev. Drug Discov. 2017, 16, 315-337. (g) Rudra, A.; Li, J.; Shakur, R.; Bhagchandani, S.; Langer, R. Trends in Therapeutic Conjugates: Bench to Clinic. Bioconjugate Chem. 2020, 31, 462-473.

(27) (a) Letschert, S.; Gohler, A.; Franke, N.; Bertleff-Zieschang, N.; Memmel, E.; Doose, S.; Siebel, J.; Sauer, M. Super-Resolution Imaging of Plasma Membrane Glycans. Angew. Chem., Int. Ed. 2014, 126, 11101-11104. (b) Chen, J.; Gao, J.; Wu, M.; Zhang, M.; Cai, H.; $\mathrm{Xu}$, J.; Jiang, Z.; Tian, Z.; Wang, H. Revealing the Carbohydrate Pattern on a Cell Surface by Super-Resolution Imaging. Nanoscale 2015, 7, 3373-3380. (c) Hegermann, J.; Lunsdirf, H.; Ochs, M.; Haller, $\mathrm{H}$. Visualization of the Glomerular Endothelial Glycocalyx by Electron Microscopy Using Cationic Colloidal Thorium Dioxide. Histochem. Cell Biol. 2016, 145, 41-51. (d) Mockl, L.; Pedram, K.; Roy, A. R.; Krishnan, V.; Gustavsson, A. K.; Dorigo, O.; Bertozzi, C. R. Quantitative Super-Resolution Microscopy of the Mammalian Glycocalyx. Dev. Cell 2019, 50, 57-72.

(28) (a) Esko, J. D.; Rostand, K. S.; Weinke, J. L. Tumor Formation Dependent on Proteglycan Biosynthesis. Science. 1988, 241, 1092 1096. (b) Matrosovich, M. N.; Matros vich, T. Y.; Gray, T.; Roberts, N. A.; Klenk, H. D. Neuraminidase in Important for the Initiation of 
Influenza Virus Infection in Human Airway Epithelium. J. Virol. 2004, 78, 12655-12667. (c) Hudak, J. E.; Canham, S. M.; Bertozzi, C. R. Glycocalyx Engineering Reveals a Siglec-Based Mechanism for NK Cell Immunoevasion. Nat. Chem. Biol. 2014, 10, 69-75. (d) Constantinou, P. E.; Morgado, M.; Carson, D. Transmembrane MucinExpression and Function in Embryo Implantation and Placentation in Regulation of Implantation and Establishment of Pregnancy in Mammals, Bazer, F. W., Geisert, R. D., Eds.; Springer: New York, NY, 2015. (e) Gandhi, J. G.; Kock, D. L.; Paszek, M. J. Equilibrium Modeling of the Mechanics and Structure of the Cancer Glycocalyx. Biophys. J. 2019, 116, 694-708.
(29) (a) Kayser, H.; Zeitler, R.; Kannicht, C.; Grunow, D.; Nuck, R.; Reutter, W. Biosynthesis of a Nonphysiological Sialic Acid in Different Rat Organs, Using $N$-Propanoyl-D-Hexosamines as Precursors. J. Biol. Chem. 1992, 267, 16934-16938. (b) Mahal, L. K.; Yarema, K. J.; Bertozzi, C. R. Engineering Chemical Reactivity on Cell Surfaces Through Oligosaccharide Biosynthesis. Science. 1997, 276, 1125-1128. (c) Saxon, E.; Bertozzi, C. R. Cell Surface Engineering by a Modified Staudinger Reaction. Science. 2000, 287, 2007-2010.

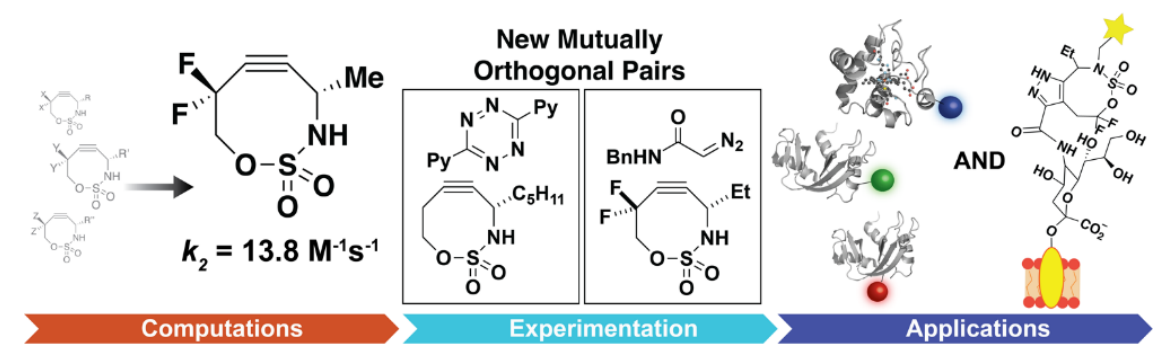

